doi: $10.2306 /$ scienceasia1513-1874.2014.40.257

\title{
Parametric inference of non-informative censored time-to-event data
}

\author{
Chris Bambey Guure ${ }^{a, *}$, Noor Akma Ibrahim ${ }^{b}$, Samuel Bosomprah ${ }^{a}$ \\ a Department of Biostatistics, School of Public Health, University of Ghana, Legon, Accra, Ghana \\ b Department of Mathematics, Faculty of Science, Universiti Putra Malaysia, Salangor, Malaysia
}

${ }^{*}$ Corresponding author, e-mail: cbguure@ug.edu.gh

Received 21 Oct 2012

Accepted 6 Nov 2013

\begin{abstract}
Random or non-informative censoring is when each subject has a censoring time that is statistically independent of their failure times. The classical approach is considered for estimating the Weibull distribution parameters with non-informative censored samples which occur most often in medical and biological study. We have also considered the Bayesian methods via gamma priors with asymmetric (general entropy) loss function and symmetric (squared error) loss function. A simulation study is carried out to assess the performances of the methods using mean squared errors and absolute biases. Two sets of data have been analysed for the purpose of illustration.
\end{abstract}

KEYWORDS: random censored data, maximum likelihood, Bayesian methods, gamma prior distribution, Weibull distribution

\section{INTRODUCTION}

Failure time analysis is used in various fields for analysing data involving the duration of events. It is also referred to as survival analysis, event history analysis, lifetime data analysis, reliability analysis or time to event analysis in different fields of study. A key characteristic that distinguishes failure time data from other areas in statistics is that failure time data are usually censored ${ }^{1}$. Censoring is said to have occurred when information about the survival time of some individuals is partially unavailable. Different conditions can bring about different types of censoring, such as, right-censoring, left-censoring and interval-censoring.

According to Horst ${ }^{2}$, "A data sample is said to be censored when, either by accident or design, the value of the variables under investigation is unobserved for some of the items in the sample". Censoring is a feature that is recurrent in lifetime and reliability data analysis, it occurs when exact lifetimes or run-outs can only be collected for a portion of the inspection units. Random or non-informative censoring is when each subject has a censoring time that is statistically independent of the failure time.

The Weibull distribution has the distribution function

$$
F(t ; \theta, p)=1-\exp \left[-\left(\frac{t}{\theta}\right)^{p}\right]
$$

the density function

$$
f(t ; \theta, p)=\frac{p}{\theta}\left(\frac{t}{\theta}\right)^{p-1} \exp \left[-\left(\frac{t}{\theta}\right)^{p}\right]
$$

and the survival function

$$
S(t ; \theta, p)=\exp \left[-\left(\frac{t}{\theta}\right)^{p}\right]
$$

where $p$ is the shape parameter and $\theta$ is the scale parameter. Research has been conducted and presented in the literature to compare the maximum likelihood estimator (MLE) and that of the Bayesian approach in estimating the parameters of the Weibull distribution. Guure $^{3}$ studied Bayesian inference of the Weibull model based on interval-censored survival data. More work can be seen in Refs. 4-6. Syuan-Rong and Shuo-Jye ${ }^{7}$ also considered Bayesian estimation and prediction for Weibull model with progressive censoring. Similar work can be seen in Shafay and Balakrishnan ${ }^{8}$, who studied one- and two-sample Bayesian prediction intervals based on type-I hybrid censored data. Gupta and Kundu ${ }^{9}$ studied generalized exponential distributions with different methods of estimation, see also Refs. 10-12, and a work on generalized exponential distribution: Bayesian estimations ${ }^{13}$, which is somehow similar to the Weibull distribution.

The aim of this paper is to consider first, the MLE of the two parameters of the Weibull distribution when 
the data under consideration are non-informatively censored. It is observed that the MLE cannot be obtained in analytical form, we therefore assumed the Newton-Raphson method to compute the MLEs, and the method works quite well. The second aim is to consider the Bayesian approach for the parameters estimates. We have considered squared error loss function which is symmetrical in nature and that of general entropy loss function which is asymmetrical. Lindley's numerical approximation procedure is employed to estimate the parameters under Bayesian.

\section{MAXIMUM LIKELIHOOD}

Let $\left(t_{1}, \ldots, t_{n}\right)$ be the set of $n$ random lifetimes with respect to the Weibull distribution with $p$ and $\theta$ as the parameters. The likelihood function with respect to non-informative/random censored data is

$$
L\left(\delta_{i}, t_{i} ; \theta, p\right)=\prod_{i=1}^{n} f\left(t_{i}, \theta, p\right)^{\delta_{i}} S\left(t_{i}, \theta, p\right)^{1-\delta_{i}},
$$

where $\delta_{i}=1$ if $X_{i} \leqslant C_{i}$, to represent uncensored observations, and $\delta_{i}=0$ if $X_{i}>C_{i}$, to represent censored observations. The observed data from $n$ individuals is assumed to consist of the pair $\left(t_{i}, \delta_{i}\right)$, $i=1,2, \ldots, n$, so that the final result obtained will be the same, provided the censoring time $C_{i}$ and the failure time $X_{i}$ are non-informative (independent of each other) and available for all $i$.

The score vectors are

$$
u(\theta)=\frac{\partial \ln L(\theta, p ; t, \delta)}{\partial \theta}, u(p)=\frac{\partial \ln L(\theta, p ; t, \delta)}{\partial p},
$$

where the score becomes a vector of the first partial derivatives of $(\theta, p)$. The score evaluated at the true parameter say, $\alpha$ has a mean zero, i.e., $E[u(\alpha)]=$ 0 and the variance-covariance matrix given as the information matrix;

$$
\operatorname{Var}[u(\alpha)]=E\left[u(\alpha) u^{\prime}(\alpha)\right]=I(\alpha) .
$$

Calculation of the maximum likelihood estimator often requires that some iterative (Newton-Raphson) procedures be implemented. We can consider evaluating MLE of $\hat{\alpha}$ with a trial value say $\alpha_{0}$ using a first order Taylor series, such that

$$
u(\hat{\alpha}) \approx u\left(\alpha_{0}\right)+\frac{\partial u(\alpha)}{\partial \alpha}\left(\hat{\alpha}-\alpha_{0}\right) .
$$

Assuming $H$ denotes the Hessian matrix, which is simply the second derivative of the log-likelihood function, then

$$
H(\alpha)=\frac{\partial^{2} \ln L}{\partial \alpha \partial \alpha^{\prime}}
$$

When the left-hand side of equation (6) is set to zero and we solve for $\hat{\alpha}$, we have

$$
\hat{\alpha}=\alpha_{0}-H^{-1}\left(\alpha_{0}\right) u\left(\alpha_{0}\right)
$$

where $u\left(\alpha_{0}\right)$ is the score vector. Using the two parameters of the Weibull distribution, the Hessian matrix can be obtained as follows. The score vector of

$u(\theta)=\frac{\partial}{\partial \theta} \ln L\left(\theta, p ; t_{i}, \delta_{i}\right)=-\frac{p}{\theta} \sum_{i=1}^{n} \delta_{i}+\frac{p}{\theta} \sum_{i=1}^{n}\left(\frac{t_{i}}{\theta}\right)^{p}$

and

$$
\begin{aligned}
u(p) & =\frac{\partial}{\partial p} \ln L\left(\theta, p ; t_{i}, \delta_{i}\right) \\
& =\frac{1}{p} \sum_{i=1}^{n} \delta_{i}+\sum_{i=1}^{n} \delta_{i} \ln \left(\frac{t_{i}}{\theta}\right)-\sum_{i=1}^{n}\left(\frac{t_{i}}{\theta}\right)^{p} \ln \left(\frac{t_{i}}{\theta}\right) .
\end{aligned}
$$

Hence we obtain the following

$$
\begin{aligned}
& \frac{\partial u(\theta)}{\partial p}=\frac{\partial u(p)}{\partial \theta} \\
&=-\frac{1}{\theta} \sum_{i=1}^{n} \delta_{i}+\frac{1}{\theta} \sum_{i=1}^{n}\left(\frac{t_{i}}{\theta}\right)^{p}+\frac{p}{\theta} \sum_{i=1}^{n}\left(\frac{t_{i}}{\theta}\right)^{p} \ln \left(\frac{t_{i}}{\theta}\right) . \\
& \frac{\partial u(\theta)}{\partial \theta}=\frac{p}{\theta^{2}} \sum_{i=1}^{n} \delta_{i}-\frac{p}{\theta^{2}} \sum_{i=1}^{n}\left(\frac{t_{i}}{\theta}\right)^{p}-\frac{p^{2}}{\theta^{2}} \sum_{i=1}^{n}\left(\frac{t_{i}}{\theta}\right)^{p} . \\
& \frac{\partial u(p)}{\partial p}=-\frac{1}{p^{2}} \sum_{i=1}^{n} \delta_{i}-\sum_{i=1}^{n}\left(\frac{t_{i}}{\theta}\right)^{p} \ln ^{2}\left(\frac{t_{i}}{\theta}\right) .
\end{aligned}
$$

The above equations give the basis for an iterative process for computing the MLEs of the parameters, which is known as Newton-Raphson approach. When a trial value is chosen carefully, (8) can be employed to estimate the parameters from which the standard errors of the parameters can be obtained.

\section{BAYESIAN INFERENCE}

In this section we consider the Bayes estimation of the two unknown parameters. Prior distribution of the parameters need to be assumed for the Bayesian inference. As discussed by Berger ${ }^{14}$ and subsequently by Banerjee and Kundu ${ }^{15}$, we let $\theta$ take on a $\gamma(a, b)$ prior distribution with $a, b>0$. We assume that the prior of $p$ is independent of the prior of $\theta$ and is in the neighbourhood of $(0, \infty)$. Let $v(p)$ represent the prior of $p$ and $v_{1}(\theta)$ for $\theta$, where

$$
\gamma(a, b) \propto \theta^{a-1} \exp (-b \theta), \quad \theta, a, b>0 .
$$

Let the likelihood equation which is $L\left(\theta, p ; t_{i}, \delta_{i}\right)$ be the same as equation (4). The joint posterior density 
function of $(\theta, p)$ is given by

$$
\pi\left(\theta, p, t_{i}, \delta_{i}\right) \propto L\left(\theta, p ; t_{i}, \delta_{i}\right) v(p) v_{1}(\theta) .
$$

The posterior probability density function of $\theta$ and $p$ given the data $\left(t_{1}, t_{2}, \ldots, t_{n}\right)$ is obtained by dividing the joint posterior density function over the marginal distribution function as

$$
\pi^{*}\left(\theta, p \mid t_{i}, \delta_{i}\right)=\frac{L\left(\theta, p ; t_{i}, \delta_{i}\right) v(p) v_{1}(\theta)}{\int_{0}^{\infty} \int_{0}^{\infty} L\left(\theta, p ; t_{i}, \delta_{i}\right) v(p) v_{1}(\theta) \mathrm{d} \theta \mathrm{d} p} .
$$

Due to the complex nature of the posterior distribution, Lindley approximation is employed in order to estimate the unknown parameters. The Bayes estimator is considered under two loss functions which is also indisputable in Bayesian estimation. They are asymmetric (general entropy) loss function and symmetric (squared error) loss function.

\section{Lindley approximation}

A prior of $p$ need to be specified here so as to calculate the approximate Bayes estimates of $\theta$ and $p$. Having specified a prior for $\theta$ as $\gamma(a, b)$, it is similarly assumed that $v(p)$ also takes on a $\gamma(c, d)$ prior. Lindley ${ }^{16}$ proposed a ratio of integral of the form

$$
\frac{\int \omega(\alpha) \mathrm{e}^{\ell(\alpha)} \mathrm{d} \alpha}{\int v(\alpha) \mathrm{e}^{\ell(\alpha)} \mathrm{d} \alpha},
$$

where $\ell(\alpha)$ is the log-likelihood and $\omega(\alpha), v(\alpha)$ are arbitrary functions of $\alpha$. Assuming that $v(\alpha)$ is the prior distribution for $\alpha$ and $\omega(\alpha)=u(\alpha) v(\alpha)$ with $u(\alpha)$ being some function of interest. Taking the Weibull two parameters into consideration, the Lindley method can be approximated asymptotically by the following

$$
\begin{aligned}
\hat{u}=u(\hat{\theta}, \hat{p}) & +\frac{1}{2}\left[\left(u_{11} \sigma_{11}\right)+\left(u_{22} \sigma_{22}\right)\right]+u_{1} \rho_{1} \sigma_{11} \\
& +u_{2} \rho_{2} \sigma_{22}+\frac{1}{2}\left[\left(\ell_{30} u_{1} \sigma_{11}^{2}\right)+\left(\ell_{03} u_{2} \sigma_{22}^{2}\right)\right],
\end{aligned}
$$

where $\ell$ is the $\log$ of the likelihood function. Hence the following derivatives are obtained first with squared error loss function;

$$
\begin{aligned}
u(\theta) & =\theta, \quad u_{1}=1, \quad u_{11}=0, \\
u(p) & =p, \quad u_{2}=1, \quad u_{22}=0, \\
\rho & =\ln v_{1}(\theta)+\ln v(p), \\
\rho_{1} & =\frac{c-1}{\alpha}-d, \quad \rho_{2}=\frac{a-1}{\beta}-b, \\
\sigma_{11} & =\left(-\ell_{20}\right)^{-1}, \quad \sigma_{22}=\left(-\ell_{02}\right)^{-1}, \\
\ell_{20} & =\frac{p}{\theta^{2}} \sum_{i=1}^{n} \delta_{i}-\frac{p^{2}}{\theta^{2}} \sum_{i=1}^{n}\left(\frac{t_{i}}{\theta}\right)^{p}-\frac{p}{\theta^{2}} \sum_{i=1}^{n}\left(\frac{t_{i}}{\theta}\right)^{p},
\end{aligned}
$$

$$
\begin{aligned}
\ell_{30}= & -\frac{2 p}{\theta^{3}} \sum_{i=1}^{n} \delta_{i}+\frac{p^{3}}{\theta^{3}} \sum_{i=1}^{n}\left(\frac{t_{i}}{\theta}\right)^{p} \\
& +\frac{3 p^{2}}{\theta^{3}} \sum_{i=1}^{n}\left(\frac{t_{i}}{\theta}\right)^{p}+\frac{2 p}{\theta^{3}} \sum_{i=1}^{n}\left(\frac{t_{i}}{\theta}\right)^{p}, \\
\ell_{02}= & -\frac{1}{p^{2}} \sum_{i=1}^{n} \delta_{i}-\sum_{i=1}^{n}\left(\frac{t_{i}}{\theta}\right)^{p} \ln ^{2}\left(\frac{t_{i}}{\theta}\right), \\
\ell_{03}= & \frac{2}{p^{3}} \sum_{i=1}^{n} \delta_{i}-\sum_{i=1}^{n}\left(\frac{t_{i}}{\theta}\right)^{p} \ln ^{3}\left(\frac{t_{i}}{\theta}\right) .
\end{aligned}
$$

\section{General entropy loss function}

The general entropy loss (GEL) function is a generalization of the entropy loss function. The Bayes estimator $\hat{\alpha}_{\mathrm{BG}}$ of $\alpha$ under the GEL is

$$
\hat{\alpha}_{\mathrm{BG}}=\left[E_{\alpha}\left(\alpha^{-k}\right)\right]^{-1 / k},
$$

provided $E_{\alpha}\left(\alpha^{-k}\right)$ exists and is finite. The Bayes estimator for this loss function is

$$
\begin{aligned}
\hat{u}_{\mathrm{BG}} & =E\left[u\left(\theta^{-k}, p^{-k}\right) \mid t\right] \\
& =\frac{\iint u\left(\theta^{-k}, p^{-k}\right) v_{1}(\theta) v(p) L\left(\delta_{i}, t_{i} ; \theta, p\right) \mathrm{d} \theta \mathrm{d} p}{\iint v_{1}(\theta) v(p) L\left(\delta_{i}, t_{i} ; \theta, p\right) \mathrm{d} \theta \mathrm{d} p} .
\end{aligned}
$$

Similar Lindley approach is used for the GEL function as in the squared error loss but here the Lindley approximation procedure where $u_{1}, u_{11}$ and $u_{2}, u_{22}$ are the first and second derivatives for $\theta$ and $p$, respectively, given below. After using the Lindley approach the function is then substituted into (12) for the parameter estimate under GEL.

$$
\begin{aligned}
u(\theta) & =\theta^{-k}, \quad u_{1}(\theta)=\frac{\partial u}{\partial \theta}=-k \theta^{-k-1}, \\
u_{11}(\theta) & =\frac{\partial^{2} u}{\partial \theta^{2}}=\left(k^{2}+k\right) \theta^{-k-2}, \\
u_{2}(\theta) & =u_{22}(\theta)=0 ; \\
u(p) & =p^{-k}, \quad u_{2}(p)=\frac{\partial u}{\partial p}=-k p^{-k-1}, \\
u_{22}(p) & =\frac{\partial^{2} u}{\partial p^{2}}=\left(k^{2}+k\right) p^{-k-2}, \\
u_{1}(p) & =u_{11}(p)=0 .
\end{aligned}
$$

\section{SIMULATION STUDY}

We have considered in this simulation study a sample size of $n=25,50$ and 100, which is representative of small, moderate and large data sets. The following steps were employed to generate the data $t_{1}, \ldots, t_{n}$. 
Table 1 Average mean squared error values for estimated parameters.

\begin{tabular}{|c|c|c|c|c|c|c|c|c|c|c|}
\hline \multirow[t]{2}{*}{ size } & \multirow[t]{2}{*}{$\theta$} & \multirow[t]{2}{*}{$p$} & \multirow[t]{2}{*}{$\hat{\theta}_{\mathrm{ML}}$} & \multirow[t]{2}{*}{$\hat{\theta}_{\mathrm{BS}}$} & \multicolumn{2}{|c|}{$\hat{\theta}_{\mathrm{GE}}$} & \multirow[t]{2}{*}{$\hat{p}_{\mathrm{ML}}$} & \multirow[t]{2}{*}{$\hat{p}_{\mathrm{BS}}$} & \multicolumn{2}{|c|}{$\hat{p}_{\mathrm{GE}}$} \\
\hline & & & & & $k=-0.7$ & $k=+0.7$ & & & $k=-0.7$ & $k=+0.7$ \\
\hline 25 & $\begin{array}{l}0.5 \\
1.5\end{array}$ & $\begin{array}{l}0.8 \\
1.2 \\
0.8 \\
1.2\end{array}$ & $\begin{array}{l}\mathbf{0 . 2 8 6 4 2} \\
\mathbf{0 . 1 1 3 3 1} \\
2.46671 \\
0.97015\end{array}$ & $\begin{array}{l}0.28654 \\
0.11339 \\
2.46775 \\
0.97088\end{array}$ & $\begin{array}{l}0.29148 \\
0.11467 \\
2.50048 \\
1.05558\end{array}$ & $\begin{array}{l}0.29560 \\
0.11687 \\
\mathbf{2 . 4 5 4 7 6} \\
\mathbf{0 . 9 3 5 4 1}\end{array}$ & $\begin{array}{l}0.02317 \\
0.04985 \\
\mathbf{0 . 0 2 1 1 0} \\
0.05326\end{array}$ & $\begin{array}{l}0.02320 \\
0.04993 \\
0.02113 \\
0.05333\end{array}$ & $\begin{array}{l}0.02286 \\
0.05236 \\
0.02303 \\
\mathbf{0 . 0 4 6 5 0}\end{array}$ & $\begin{array}{l}\mathbf{0 . 0 2 1 5 8} \\
\mathbf{0 . 0 4 8 2 3} \\
0.02160 \\
0.04960\end{array}$ \\
\hline 50 & $\begin{array}{l}0.5 \\
1.5\end{array}$ & $\begin{array}{l}0.8 \\
1.2 \\
0.8 \\
1.2\end{array}$ & $\begin{array}{l}0.19128 \\
0.08377 \\
\mathbf{1 . 5 9 4 0 0} \\
0.79669\end{array}$ & $\begin{array}{l}0.19129 \\
0.08378 \\
1.59408 \\
0.79676\end{array}$ & $\begin{array}{l}\mathbf{0 . 1 8 2 9 2} \\
0.08487 \\
1.69874 \\
\mathbf{0 . 7 6 3 2 1}\end{array}$ & $\begin{array}{l}0.19062 \\
\mathbf{0 . 0 8 3 0 9} \\
1.62575 \\
0.76659\end{array}$ & $\begin{array}{l}0.00910 \\
0.02186 \\
0.00928 \\
0.02248\end{array}$ & $\begin{array}{l}0.00910 \\
0.02187 \\
\mathbf{0 . 0 0 9 2 7} \\
0.02249\end{array}$ & $\begin{array}{l}0.00920 \\
0.02043 \\
0.00946 \\
\mathbf{0 . 0 1 9 5 5}\end{array}$ & $\begin{array}{l}\mathbf{0 . 0 0 8 4 4 4} \\
\mathbf{0 . 0 1 9 5 0} \\
0.00961 \\
0.02233\end{array}$ \\
\hline 100 & $\begin{array}{l}0.5 \\
1.5\end{array}$ & $\begin{array}{l}0.8 \\
1.2 \\
0.8 \\
1.2\end{array}$ & $\begin{array}{l}0.15350 \\
0.07239 \\
1.41109 \\
0.67386\end{array}$ & $\begin{array}{l}0.15350 \\
0.07239 \\
1.41109 \\
0.67387\end{array}$ & $\begin{array}{l}0.15116 \\
\mathbf{0 . 0 7 2 3 4} \\
1.38946 \\
0.64008\end{array}$ & $\begin{array}{l}\mathbf{0 . 1 4 5 4 4} \\
0.07275 \\
\mathbf{1 . 3 8 6 6 3} \\
\mathbf{0 . 6 3 6 3 4}\end{array}$ & $\begin{array}{l}0.00423 \\
\mathbf{0 . 0 0 9 2 2} \\
\mathbf{0 . 0 0 4 2 7} \\
0.00950\end{array}$ & $\begin{array}{l}0.00423 \\
\mathbf{0 . 0 0 9 2 2} \\
\mathbf{0 . 0 0 4 2 7} \\
0.00950\end{array}$ & $\begin{array}{l}0.00440 \\
0.00951 \\
0.00443 \\
\mathbf{0 . 0 0 9 0 0}\end{array}$ & $\begin{array}{l}\mathbf{0 . 0 0 4 0 9} \\
0.00968 \\
0.00435 \\
0.01018\end{array}$ \\
\hline
\end{tabular}

$\mathrm{ML}=$ Maximum likelihood, $\mathrm{BS}=$ Bayes squared error loss, $\mathrm{GE}=$ Bayes general entropy loss.

The smallest value in each group is marked in bold face.

A lifetime $t_{i}$ is generated from the sample sizes indicated above from the Weibull distribution which represents failure of the product or unit. The values of the assumed actual parameters of the Weibull distribution were taken to be $\theta=0.5$ and 1.5 and that of $p=0.8$ and 1.2. The same sample size is generated from the Uniform distribution for the censored time $C_{i}$ with $(0, b)$, where $b$ depends solely on the proportion of the observations that are censored. In our study, we consider the percentage of censoring to be 40 . The $t_{i}$ is taken as the minimum of the failure time and the censored time, $t_{i}:=\min \left(X_{i}, C_{i}\right)$, and where $\delta_{i}=1$ if $X_{i} \leqslant C_{i}$ for uncensored observations, and $\delta_{i}=0$ if $X_{i}>C_{i}$ for censored observations.

To compute the Bayes estimates, an assumption is made such that $\alpha$ and $\beta$ take, respectively, $\gamma(a, b)$ and $\gamma(c, d)$ priors. We set the hyper-parameters to 0.0001 , i.e., $a=b=c=d=0.0001$ in order to obtain noninformative priors. Note that at this point the priors become proper as well as the posterior distribution, Press ${ }^{17}$

The values for the loss parameter for the general entropy was $k= \pm 0.7$, without loss of generality. For further discussions on the choice of the loss parameters, see Refs. 18, 19. These were iterated 1000 times. The mean squared error (MSE) and the absolute bias values are determined and presented below for the purpose of comparison.

\section{RESULTS AND DISCUSSION}

Bayes estimators of the Weibull parameters are obtained using squared error and general entropy loss functions by employing Lindley approximation procedure. Comparisons are made between the estimators using MSE and absolute bias based on simulation study. The performance of MLE and Bayes using the gamma non-informative priors were examined and the following observations made.

Table 1 shows the MSE values of the scale and shape parameters. It is been observed that Bayes estimator under the general entropy loss function has the smallest MSE values than the others. This occurred mostly with the positive loss parameter, i.e., +0.7 . This is followed by both the maximum likelihood estimator and Bayes estimator under general entropy with -0.7 as the loss parameter. Both obtained equal estimate for the scale parameter but for the shape parameter, maximum likelihood was slightly ahead of Bayes with general entropy of -0.7 .

The absolute bias of the estimated values are presented in Table 2. We observe again that Bayes using the general entropy loss function with +0.7 outperformed Bayes using squared error and general entropy of -0.7 , and that of the classical maximum likelihood estimator for both parameters. This was followed by maximum likelihood, general entropy of -0.7 and Bayes estimator under squared error loss function, respectively, for the shape parameter. For the scale parameter, we observed that after general entropy loss function with +0.7 , general entropy of -0.7 outperformed the others. All the estimators had their MSEs decreasing with an analogous increase in sample space. As the sample size increased, maximum likelihood and Bayes estimator under squared error loss function tern to converge to the same values with respect to the MSE and absolute bias values for both parameters

For each estimated parameter, the MSE is calculated for each of the one thousand estimated values of the parameters, i.e., from 1-1000. For instance, $r=1$ has an estimate for both the scale parameter and the shape parameter and so the MSE of these 
Table 2 Average absolute bias values for estimated parameters.

\begin{tabular}{|c|c|c|c|c|c|c|c|c|c|c|}
\hline \multirow[t]{2}{*}{ size } & \multirow[t]{2}{*}{$\theta$} & \multirow[t]{2}{*}{$p$} & \multirow[t]{2}{*}{$\hat{\theta}_{\mathrm{ML}}$} & \multirow[t]{2}{*}{$\hat{\theta}_{\mathrm{BS}}$} & \multicolumn{2}{|c|}{$\hat{\theta}_{\mathrm{GE}}$} & \multirow[t]{2}{*}{$\hat{p}_{\mathrm{ML}}$} & \multirow[t]{2}{*}{$\hat{p}_{\mathrm{BS}}$} & \multicolumn{2}{|c|}{$\hat{p}_{\mathrm{GE}}$} \\
\hline & & & & & $k=-0.7$ & $k=+0.7$ & & & $k=-0.7$ & $k=+0.7$ \\
\hline 25 & $\begin{array}{l}0.5 \\
1.5\end{array}$ & $\begin{array}{l}0.8 \\
1.2 \\
0.8 \\
1.2\end{array}$ & $\begin{array}{l}0.41260 \\
0.27453 \\
1.21538 \\
0.80012\end{array}$ & $\begin{array}{l}\mathbf{0 . 4 1 2 5 9} \\
0.27464 \\
1.21566 \\
0.80045\end{array}$ & $\begin{array}{l}0.41860 \\
\mathbf{0 . 2 7 4 1 9} \\
1.18024 \\
0.83827\end{array}$ & $\begin{array}{l}0.41267 \\
0.27636 \\
\mathbf{1 . 1 7 8 7 6} \\
\mathbf{0 . 7 7 7 2 8}\end{array}$ & $\begin{array}{l}0.11246 \\
0.16723 \\
\mathbf{0 . 1 0 9 3 7} \\
0.17492\end{array}$ & $\begin{array}{l}0.11251 \\
0.16732 \\
0.10943 \\
0.17501\end{array}$ & $\begin{array}{l}0.11520 \\
0.17256 \\
0.11355 \\
\mathbf{0 . 1 6 7 6 0}\end{array}$ & $\begin{array}{l}\mathbf{0 . 1 1 2 2 5} \\
\mathbf{0 . 1 6 4 1 0} \\
0.11277 \\
0.16832\end{array}$ \\
\hline 50 & $\begin{array}{l}0.5 \\
1.5\end{array}$ & $\begin{array}{l}0.8 \\
1.2 \\
0.8 \\
1.2\end{array}$ & $\begin{array}{l}0.36507 \\
0.25442 \\
1.09198 \\
0.78558\end{array}$ & $\begin{array}{l}0.36508 \\
0.25443 \\
1.09201 \\
0.78561\end{array}$ & $\begin{array}{l}\mathbf{0 . 3 5 7 5 1} \\
\mathbf{0 . 2 5 4 2 5} \\
1.09955 \\
0.77135\end{array}$ & $\begin{array}{l}0.37268 \\
0.25370 \\
\mathbf{1 . 0 7 6 7 9} \\
\mathbf{0 . 7 6 5 5 2}\end{array}$ & $\begin{array}{l}0.07444 \\
0.11673 \\
\mathbf{0 . 0 7 3 8 5} \\
0.11508\end{array}$ & $\begin{array}{l}0.07445 \\
0.11674 \\
0.07386 \\
0.11511\end{array}$ & $\begin{array}{l}0.07454 \\
0.11021 \\
0.07581 \\
\mathbf{0 . 1 0 9 6 5}\end{array}$ & $\begin{array}{l}\mathbf{0 . 0 7 3 3 8} \\
\mathbf{0 . 1 1 0 0 7} \\
0.07702 \\
0.11522\end{array}$ \\
\hline 100 & $\begin{array}{l}0.5 \\
1.5\end{array}$ & $\begin{array}{l}0.8 \\
1.2 \\
0.8 \\
1.2\end{array}$ & $\begin{array}{l}0.35707 \\
\mathbf{0 . 2 5 0 2 0} \\
1.07443 \\
0.76893\end{array}$ & $\begin{array}{l}0.35707 \\
\mathbf{0 . 2 5 0 2 0} \\
1.07443 \\
0.76893\end{array}$ & $\begin{array}{l}0.35132 \\
0.25202 \\
1.07292 \\
0.74494\end{array}$ & $\begin{array}{l}\mathbf{0 . 3 4 5 3 4} \\
0.25101 \\
\mathbf{1 . 0 5 9 4 9} \\
\mathbf{0 . 7 3 8 6 3}\end{array}$ & $\begin{array}{l}0.05155 \\
\mathbf{0 . 0 7 4 9 2} \\
\mathbf{0 . 0 5 1 2 2} \\
0.07701\end{array}$ & $\begin{array}{l}0.05156 \\
\mathbf{0 . 0 7 4 9 2} \\
0.05123 \\
0.07701\end{array}$ & $\begin{array}{l}0.05242 \\
0.07648 \\
0.05219 \\
\mathbf{0 . 0 7 4 1 1}\end{array}$ & $\begin{array}{l}\mathbf{0 . 0 5 0 9 1} \\
0.07584 \\
0.05192 \\
0.07875\end{array}$ \\
\hline
\end{tabular}

$\mathrm{ML}=$ Maximum likelihood, $\mathrm{BS}=$ Bayes squared error loss, $\mathrm{GE}=$ Bayes general entropy loss.

The smallest value in each group is marked in bold face.

two are taken, also $r=2$ also has scale parameter and the shape parameter estimates where the MSE are calculated, it continues in like manner for all $r=1000$ of all the estimates. At the end, we obtain the average of the MSE values. Our aim is to find out how close the estimated values of the estimators are to the true values at each simulated point. The absolute bias values are obtained in like manner and of course from the same simulated values as that of the MSE.

\section{REAL DATA ANALYSIS}

Example 1 In this section, we analyse one data set to obtain the parameter estimates and their standard errors in order to compare the estimators assumed in this paper. The data below are obtained from Lawless $^{20}$ about remission times, in weeks, for a group of 30 patients with leukaemia who received similar treatment (asterisks denote censoring times): $1,1,2,4,4,6,6,6,7,8,9,9,10,12,13,14,18,19$, $24,26,29,31^{*}, 42,45^{*}, 50^{*}, 57,60,71^{*}, 85^{*}, 91$.

Using the iterative procedure suggested from the beginning of this paper, the MLEs of $\hat{\theta}$ and $\hat{p}$ can be obtained as 28.27991 and 0.99201 , respectively, with their corresponding standard errors as 1.56748 and 0.02386 . Since we do not have any prior information on the hyper-parameters, we assume $a=b=c=$ $d=0.0001$. This makes the priors proper on $\hat{\theta}$ and $\hat{p}$, and the corresponding posteriors also proper.

When we compute the Bayes estimators under squared error loss of $\hat{\theta}$ and $\hat{p}$, the following parameters estimates and standard errors are obtained, respectively, as 28.32146, 0.99201 and 1.56980, 0.02386 . When we compute the Bayes estimates of $\hat{\theta}$ and $\hat{p}$ and their corresponding standard errors under the general entropy loss functions with the loss parameter being -0.7 we have $28.57414,1.10030$ and 1.57112 ,
0.02467. With the loss parameter being +0.7 , we have $28.14560,0.82215$ and $1.49185,0.02286$, respectively.

Observably, the Bayes estimator under squared error loss for the Weibull shape parameter $(p)$ has the same estimate and standard error as compared to that of the classical maximum likelihood estimator but with the scale parameter $(\hat{\theta})$, maximum likelihood has a smaller standard error in juxtaposition to Bayes estimator under squared error loss.

Comparing all the estimators, it is clear from the results that Bayes estimator under general entropy loss function with the loss parameter of +0.7 has the smallest standard error and estimate for both the shape parameter $\hat{p}$ and the scale parameter $\hat{\theta}$.

Example 2 The data for this example are on survival of patients with cervical cancer, recruited to a randomised trial aimed at analysing the effect of addition of a radio sensitiser to radiotherapy (New therapy "treatment B") compared to using only radiotherapy (Control - "treatment A"). Treatment A was given to 16 and treatment B to 14 patients. The data are in days since start of the study, the event of interest is death caused by this cancer. Our main interest is on the patients under treatment $\mathrm{B}$, which is fairly small and highly censored to illustrate the proposed methods in this paper. The data are obtained from Coolen and Yan $^{21}$ (starred observations are censored): 272, 362, 373, 383*, 519*, 563*, 650*, 827, 919*, 978*, 1100*, 1307, 1360*, 1476*.

The MLEs of $\hat{\theta}$ and $\hat{p}$ are 1374.7080 and 2.12549 with their corresponding standard errors as 79.5806 and 0.21954 , respectively.

The Bayes estimators under squared error loss of $\hat{\theta}$ and $\hat{p}$, has, respectively, the following parameters estimates and standard errors as 1380.3460, 2.12564 
and $83.2868,0.21958$

Computing the Bayes estimates of $\hat{\theta}$ and $\hat{p}$ and that of their standard errors via the general entropy loss function with a loss parameter of -0.7 we have, $1379.8070,2.12556$ and 82.9167, 0.21958. With the loss parameter of +0.7 , we have, $1377.2800,2.12519$ and $81.0723,0.21955$, respectively.

What we observed from this fairly small sample is that maximum likelihood has among the other estimators the smallest standard error for the scale parameter but for the shape parameter both maximum likelihood and Bayes under the positive general entropy loss parameter had the smallest standard error for the Weibull distribution.

When we take into perspective a $95 \%$ confidence interval of MLE for the fairly small sample, we have $\hat{\theta}=(1530.6860,1218.7300)$ and $\hat{p}=(2.5558$, 1.6952). Bayes credible intervals under squared error loss function for $\hat{\theta}$ and $\hat{p}$ are $(1543.5881,1217.1039)$ and $(2.5560,1.6953)$, respectively. The Bayes credible intervals with respect to the general entropy loss function with a loss parameter of -0.7 for $\hat{\theta}$ and $\hat{p}$ are $(1542.3237,1217.2903)$ and $(2.5559,1.6952)$ and that of the +0.7 are $(1536.1817,1218.3783)$ and $(2.5551$, 1.6949), respectively.

Observing from above, it is clear that general entropy with a positive loss parameter and maximum likelihood had narrower credible and confidence intervals, respectively, as compared to squared error loss function for the shape parameter. For the scale parameter, maximum likelihood's confidence interval is narrower than the Bayesian credible intervals.

\section{CONCLUSIONS}

From the above discussions, in relation to both the real data analysis and the simulation study, we conclude that the best estimator of the two parameter Weibull distribution with this type of censoring, when the data is fairly moderate or large will be Bayes estimator under general entropy loss function provided the loss parameter is well chosen. For fairly small sample, maximum likelihood can be a good alternate to the Bayes estimators.

\section{REFERENCES}

1. Gómez G, Calle ML, Oller R (2004) Frequentist and Bayesian approaches for interval-censored data. Stat Paper 45, 139-73.

2. Horst R (2009) The Weibull Distribution Handbook. CRC Press, Taylor and Francis Group LLC, New York.

3. Guure CB, Ibrahim NA, Adam MB (2013) Bayesian inference of the Weibull model based on intervalcensored survival data. Comput Math Meth Med 2013, Article ID 849520, doi: 10.1155/2013/849520.
4. Sinha SK (1986) Bayes estimation of the reliability function and hazard rate of a Weibull failure time distribution. Trab Estad 1, Issue 2, 47-56.

5. Lye LM, Hapuarachchi KP, Ryan S (2010) Bayes estimation of the extreme-value reliability function. IEEE Trans Reliab 42, 641-4.

6. Guure CB, Ibrahim NA (2012) Bayesian analysis of the survival function and failure rate of Weibull distribution with censored data. Math Probl Eng 2012, Article ID 329489, doi: 10.1155/2012/329489.

7. Huang SR, Wu SJ (2011) Bayesian estimation and prediction for Weibull model with progressive censoring. J Stat Comput Simulat 82, 1607-20.

8. Shafay AR, Balakrishnan N (2012) One- and twosample Bayesian prediction intervals based on type-I hybrid censored data. Comm Stat Simulat Comput 41, 65-88.

9. Gupta RD, Kundu D (2000) Generalized exponential distributions: different methods of estimation. J Stat Comput Simulat 69, 315-38.

10. Zellner A (1986) Bayesian estimation and prediction using asymmetric loss functions. J Am Stat Assoc 81, 446-51.

11. Al-Aboud FM (2009) Bayesian estimation for the extreme value distribution using progressive censored data and asymmetric loss. Int Math Forum 4, 1603-22.

12. Al-Athari FM (2011) Parameter estimation for the double-pareto distribution. J Math Stat 7, 289-94.

13. Kundu D, Gupta RD (2008) Generalized exponential distribution: Bayesian estimations. Comput Stat Data Anal 52, 1873-83.

14. Berger JO, Sun D (1993) Bayesian analysis for the Poly-Weibull distribution. J Am Stat Assoc 88, 1412-8.

15. Banerjee A, Kundu D (2008) Inference based on type-II hybrid censored data from a Weibull distribution. IEEE Trans Reliab 57, 369-78.

16. Lindley DV (1980) Approximate Bayesian methods. Trab Estad Investig Oper 31, 223-37.

17. Press SJ, Tanur JM (2001) The Subjectivity of Scientists and the Bayesian Approach, Wiley, New York.

18. Guure CB, Ibrahim NA, Adam MB, Ahmed AOM, Bosomprah S (2014) Bayesian parameter and reliability estimate of Weibull failure time distribution. Bull Malays Math Sci Soc 37, 611-32.

19. Calabria R, Pulcini G (1996) Point estimation under asymmetric loss functions for left-truncated exponential samples. Comm Stat Theor Meth 25, 585-600.

20. Lawless JF (2003) Statistical Models and Methods for Lifetime Data. Wiley, New York.

21. Coolen FPA, Yan KJ (2004) Nonparametric predictive inference with right-censored data. J Stat Plann Infer 126, 25-54. 